\title{
Modelo de Gestión "Business Process Management" para mejorar los Resultados del Centro de Salud de Morales - San Martín, 2020
}

Lizeth Erly Mescua Ampuero lizing20@gmail.com Universidad César Vallejo ORCID: $\underline{0000-0003-2748-479 X}$

Evangelina Ampuero Fernández ampueroevangelina@hotmail.com Universidad Nacional de San Martín ORCID: 0000-0003-2952-8354

José Manuel Delgado Bardales jmdelgadob@ucvvirtual.edu.pe

Universidad César Vallejo

ORCID:이이-0001-6574-2759

\section{RESUMEN}

El presente trabajo se caracteriza principalmente por caracterizar la gestión por procesos para mejorar los resultados de gestión en el Centro de Salud de Morales - San Martín 2020. La investigación se sostiene en los aportes teóricos que ayudarán al reforzamiento del marco teórico existente. El objetivo es Proponer un Modelo de Gestión "Business Process Management" para mejorar los Resultados del Centro de salud de Morales de la Red San Martín, 2020. Se recurrió a la investigación aplicada de enfoque cualitativo, de diseño de investigación no experimental, descriptivo, propositivo. Como técnicas de recolección de datos se ha empleado la encuesta y como instrumento cuestionarios por cada variable de estudio. Se concluyó que la gestión por procesos BPM es una herramienta de mejora de la gestión más efectivas para el Centro de Salud de Morales ; de aquí, se desprende que existe una necesidad por parte de la institución de aplicar y ejecutar estrategias de procesos gerenciales con miras a alcanzar niveles óptimos de calidad, apoyados en herramientas modernas que se adapten a las necesidades actuales del entorno., es decir a que la mejora de procesos, conlleva a simplificación administrativa y la satisfacciòn de los usuarios del Centro de Salud de Morales, siendo lo más resaltante la socializaciòn de los instrumnentos de gestión , resultados de gestión, capacitaciones y seguimiento de los procesos de cada área.

Palabras clave: Gestión, procesos, Gestión pública, administración. 


\title{
Management model "Business Process Management" to improve the Results of the Morales Health Center - San Martín, 2020
}

\begin{abstract}
:
This work is mainly characterized by characterizing management by processes to improve management results. The research is based on the theoretical contributions that will help to reinforce the existing theoretical framework. The objective is to Propose a Management Model "Business Process Management" to improve the Results of the Morales Health Center of the San Martín Network, 2020. Applied research with a qualitative approach, non-experimental, descriptive research design was used. purposeful. As data collection techniques the survey has been used and questionnaires for each study variable have been used as an instrument. It was concluded that management by BPM processes is a more effective management improvement tool for the Morales Health Center; Hence, it follows that there is a need for the institution to apply and execute management process strategies with a view to achieving optimal levels of quality, supported by modern tools that adapt to the current needs of the environment, that is, The improvement of processes leads to administrative simplification and the satisfaction of the Morales Health Center users, the most outstanding being the socialization of the management instruments, management results, training and monitoring of the processes of each area.
\end{abstract}

Keywords: Management, processes, Public management, administration

Artículo recibido: 21 oct. 2020 Aceptado para publicación: 07 nov. 2020 Correspondencia: lizing20@gmail.com. Conflictos de Interés: Ninguna que declarar 


\section{INTRODUCCIÓN}

El mundo viene evolucionando a gran velocidad, permitiendo que surjan nuevos retos y oportunidades para las organizaciones en calidad, tecnología y administración, por ello, durante los últimos períodos la tendencia es lograr productos y servicios de calidad centrado en las necesidades del usuario. En ese sentido, el sector salud, principal empleador del sector de servicios se encuentra en el 7 y $13 \%$ de la mano de obra, según reporta la Organización Mundial de la Salud [OMS], 2009 - 2014. Entre 2000 y 2006, hubo un incremento de participación en el producto interno bruto (PIB), al pasar del 8,0\% al 8,7\%, existiendo una mayor necesidad de especialista de la salud ante la existencia de una pandemia (OMS, 2019).

Según el estudio realizado por el Banco interamericano de desarrollo, Brasil, Chile, Colombia y México poseen innovaciones gerenciales; de 25 países estudiados, 19 de ellos se encuentran en fase de propuesta o implementación inicial de planificación orientados a resultados. El elemento más crítico es la debilidad de las estrategias de mejoramiento de la calidad de los bienes y servicios, definido dentro de la piedra angular de la gestión por resultados.

La reforma de la administración pública peruana durante la década de los 90, criticaba el carácter autoritario y la concentración del poder de decisiones con el que venían funcionando las instituciones (Prats, 1992, 88), buscaba reorganizar las entidades del gobierno central, gobiernos regionales, instituciones públicas descentralizadas, corporaciones de desarrollo y proyectos especiales. Desde entonces la promulgación de de la Política de Modernización de la Gestión Pública al 2021 (D.S. N 004-2013-PCM), representa un importante punto de inflexión y gestión de cambio, a partir de la cual se replantearon en términos de calidad de servicio e innovación administrativa, centrado en las necesidades del usuario, la forma en que se gestionan las instituciones públicas. Se trata del inicio de una gestión orientada a una "organización por procesos", con la finalidad de generar valor a los bienes y servicios, para ello se debe establecer la visión, los instrumentos de gestión para una actuación organizada, coherente y eficaz del sector público(Escobar, 2017, p. 3)

En ese sentido, la gestión y mejora de procesos resulta una de las buenas prácticas reconocidas, en la actualidad, en el campo de la gestión organizacional, debido a que el mundo ha evolucionado permitiendo que surjan nuevos retos y oportunidades de calidad, tecnología y administración. Por ello las organizaciones enfrentan la necesidad de mejorar la calidad del producto o servicio que brindan u ofertan.

En nuestro país, el tercer eje de la Política de Modernización establece que la gestión por procesos promueve una gestión pública con enfoque de servicio. Por ello, es importante que las instituciones orienten su gestión hacia una "organización por procesos", con la finalidad de 
generar valor a los bienes y servicios (Escobar, 2017, p. 3), para ello se debe establecer la visión, los instrumentos de gestión para una actuación organizada, coherente y eficaz del sector público.

Los ministerios sectoriales y las instituciones cuentan con sistemas de información de monitoreo de la gestión por resultados o indicadores de desempeño para la evaluación del gasto. En el sector existe la unidad de estadística que dispone de información sobre los servicios que se atienden. Sin embargo la información tiene un retraso de tiempo, carece de información de calidad debido a la falta de estándares en sus procesos para analizar eficiencia de las acciones que realizan.

El tercer pilar de la PNMGP es la Gestión por Procesos, Simplificación Administrativa y Organización Institucional.” Por ende, las organizaciones públicas, buscan formas de cómo organizar, formalizar y automatizar todos sus procesos, con la finalidad de tener controlado toda su cadena de valor.

En ese marco, en la actualidad frente a la pandemia del COVID 19, el sistema de salud actual en la región San Martín ha presentado deficiencia en la atención de los servicios de salud a la ciudadanía, debido a que los insumos, materiales y/o, equipos en los servicios han sido insuficientes para poder cubrir la demanda creciente ocasionado por esta enfermedad. Tal es así, que el gobierno peruano ha tenido que declarar el estado de emergencia nacional (D.S. 044-2020PCM), para mitigar o estabilizar el número de pacientes que requieran atención hospitalaria, afectando el desarrollo de actividades de diversos sectores, y más aún en el sector salud, donde pacientes que requieren atención médica hospitalaria y exámenes de diagnóstico de la enfermedad, no pudieron realizarlo, por la carencia de éstos en los servicios o por la prioridad en su realización por pacientes con enfermedades más graves. Por lo tanto, pudo haber un gran número de casos sin detectar entre los pacientes que acudieron a centros de primer nivel de atención o que no han buscado atención (Ministerio de Salud, 2020, p. 2).

Tal es así, que las instituciones de la salud orientan la organización hacia un enfoque de gestión por procesos, definiendo los mapas de procesos que responde a nuevas estrategias para estandarizar los procesos, ejecutar estrategias y fomentar un modelo de trabajo colaborativo, con enfoque de sistemas, trabajo colaborativo y ofreciendo una visión transversal para garantizar la sostenibilidad(Aliaga, 2015, p. 1); pero esto ha quedado en escritorio y su implementación aún está distante o se ha realizado en un bajo porcentaje, no evidenciando cambios positivos en los indicadores de salud.

El Centro de Salud de Morales - Red San Martín ubicado en el distrito de Morales, con una población microred 2016: 34,464 habitantes, con un incremento del 1.4\% de crecimiento poblacional al año y número de establecimientos : 2 Centros de Salud y 2 Puestos de Salud. El Centro de Salud de Morales de la microred Morales registra un total de 138 trabajadores, el mismo que representa el $21.3 \%$ del total del recurso humano de la REDSM, solo el $40.5 \%$ tiene 
la condición de nombrado de planta y la diferencia contratados(Médicos 9.2\%, Obstetras 8.6\%, Enfermeras $9.2 \%$, Odontólogos 3.5\% y personal técnico representa aproximadamente el $57.1 \%$ ) Actualmente en el Centro de Salud de Morales - Red San Martín, existe deficiencia en la gestión administrativa , trabajan de manera funcional, existe desconocimiento de actividades a realizar, no llevan una adecuada planificación estratégica de lo que quiere lograr afectando los resultados e indicadores de gestión de los servicios de la salud y la calidad del servicio adecuado para la población de San Martín.

En este contexto es importante que la institución Centro de salud de Morales de la Red San Martín, implemente la gestión por procesos para automatizar y optimizar el tiempo de respuesta para lograr buenos resultados. En ese sentido, el presente trabajo de investigación permitió diseñar un modelo de Gestión por procesos para mejorar los Resultados del Centro de salud de Morales de la Red San Martín.

Ante esta problemática no planteamos la siguiente interrogante ¿Cómo el Modelo de Gestión "Business Process Management" puede mejorar los Resultados del Centro de salud de Morales de la Red San Martín, 2020?

Así mismo la Justificación del estudio radica en que la investigación permitió brindar el Modelo de Gestión por procesos basado en bussiness process management para mejorar los resultados de la gestión del Centro de salud de Morales de la Red San Martín y obtener una mejora organizacional, para ello debemos tener en cuenta la sistematización definitiva de la información, métodos y evidencia, según Donabedian (año, p60.).

Por esto, desde el punto de vista metodológico, se establecerá los criterios para la caracterización y gestión sistemática de las actividades, la relación que existe entre tales procesos y los resultados del Centro de Salud de Morales en la buena calidad de servicio de atención médica a los usuarios en el Centro de Salud del distrito de Morales; cabe señalar que, en la presente investigación, la situación problemática mencionada corresponde a los años 2019 - 2020. Asimismo, valorar los aportes del presente trabajo, que se apoya en bases teóricas y metodológicas, considerando el método, instrumentos para la recolección de datos y análisis de resultados. Contribuyó en resolver los problemas y limitaciones del centro de salud, así como mejorar el servicio que brindan a los pacientes. Con el presente estudio se diseñó nuevos instrumentos de recopilar información.

Por ello, nos planeamos el siguiente objetivo general: Proponer un Modelo de Gestión "Business Process Management" para mejorar los Resultados del Centro de salud de Morales de la Red San Martín, 2020. Y como objetivos específicos: i). Caracterizar la gestión actual del Centro de Salud de Morales, basado en el DS 004-2013/PCM; ii). Evaluar los procesos de gestión del centro de salud de Morales; iii) Diseñar el modelo de gestión por procesos basado en la metodología BPM para mejorar los resultados del Centro de Salud de Morales de la Red San Martín iv). Validar el 
modelo de gestión a través de juicio de expertos. Y como hipótesis nos planteamos: El Modelo de Gestión basada en la modernización de la gestión pública y en la metodología de "Business Process Management" mejorará los Resultados del Centro de salud de Morales de la Red San Martín, 2020.

Con el propósito de sustentar la investigación se muestra estudios ya realizados en relación a la variable, considerando en el contexto internacional a la investigación de Barrios, K., Contreras, J. (2019) en su investigación La gestión por procesos en las PYMES de Barranquilla: Factor diferenciador de la competitividad organizacional. (artículo científico). Colombia. Estudio cualitativo no experimental, evaluó el impacto del modelo en la organización de pequeñas y medianas empresas del sector comercio y servicio; población y muestra estuvo constituida por 299 empresas en el país, utilizó el análisis documental y la ficha de recolección de datos como instrumento. Concluye fortalecer el ecosistema y compromiso de los sectores involucrados, instituciones y empresas y otros agentes del entorno, para la aplicación de mecanismos de mejora. Por su parte, Ramos, J., García, J., Cid, V. (2018) en su investigación Diseño de un marco de trabajo dirigido por modelos y soportado por herramientas para mejorar la gestión de guías clínicas y procesos asistenciales. (artículo científico). Universidad de Sevilla, España. Investigación propositivo, evaluó la implementación de un sistema de gestión de guías clínicas, validada por profesionales especialistas del centro hospitalario. Utilizó la revisión documental como técnica y la ficha de recolección de datos de publicaciones científicas. Concluye que en el proceso de pruebas se han adecuado de acuerdo a los requerimientos de profesionales sanitarios y que adicional está siendo evaluada con aproximadamente 20 pacientes con Diabetes Mellitus Tipo 2

Asimismo, Medina, A. (2018) en su investigación Procedimiento para la gestión por procesos: métodos y herramientas de apoyo. (artículo científico) Universidad de Matanzas Cuba. Estudio descriptivo. Población y muestra estuvo conformado por 40 empresas de manufactura y servicio, propone una mejora de procesos con enfoque al cliente, el alineamiento estratégico y la mejora continua. Utilizó la revisión documental como técnica y la ficha de recolección de datos de publicaciones científicas. Concluye que se debe priorizar los procesos que responden a los objetivos de las organizacione para lograr un impacto positivo en los clientes.

Al respecto, Hernández, A., Delgado, A. (2016) en su investigación Generalización de la gestión por procesos como plataforma de trabajo de apoyo a la mejora de organizaciones de salud (artículo científico). Universidad de Matanzas Camilo Cienfuegos, Cuba. Estudio Cuantitativo, aplicó la ficha de recolección de datos de publicaciones aplicadas a artículos científicos de revistas indexadas. Población y/o muestra, estuvo conformada por cinco hospitales de una provincia cubana para evaluar la utilidad de los instrumentos y posterior apreciar los avances en el servicio 
y el desempeño hospitalario. Concluye que la aplicación de herramientas de gestión por procesos mejora los resultados en utilidad y pertinencia logrando calidad del servicio que brindan.

Asimismo, Pascual, C. (2015) en su investigación Definición y estabilización de los procesos I, procesos de gestión y soporte en un servicio de Urología (artículo científico) Madrid (España); evalúa los procesos clínicos basado en procedimentos transuretrales sin complicaciones. Utilizó como técnica el análisis documento y como instrumento la ficha de recolección de datos de publicaciones. Concluye que los planes de mejora con enfoque de procesos permiten establecer procesos clínicos en términos de volumen de actividad.

Asimismo, en cuanto a los trabajos previos realizados a nivel nacional, se menciona a Navarro, N. (2017), en su investigación: Gestión por procesos y su relación con la calidad de servicio en el área de Obstetricia del Centro de Salud del distrito de Andahuaylas, 2016 (Trabajo de investigación) Universidad Nacional José María Arguedas, estudio no experimental transeccional - correlacional; técnica la encuesta y como instrumento el cuestionario. Concluye que a un mayor incremento de elementos tangibles, empatía, seguridad, fiabilidad frente al mismo comportamiento en la estructura, procesos y resultados en la gestión, se incrementará la mejora en el servicio de salud en el área de Obstetricia.

En referencia a los trabajos previos realizados a nivel local, se recoge el aporte de Santa María T.(2018), en su investigación Gestión por procesos y su relación con la asistencia técnica contable para los usuarios del Centro de Servicios de Atención al Usuario - CONECTAMEF en el departamento de San Martín - 2018 (Tesis de maestría) Universidad César Vallejo, estudio no experimental, descriptivo y transversal de relación causal; los actores de estudio estuvieron conformado por un total de 67 representantes del área contable, utilizó como la encuesta y el cuestionario para la recolección de datos. Concluye que la gestión por procesos y la asistencia técnica contable tiene una relación significativa.

Así también, Vallejos, V. (2018) en su investigación Sistema de Gestión de Calidad según ISO 9001:2015 para mejorar procesos de capacitación, Universidad Nacional de San Martín Tarapoto, 2018 (tesis doctoral). San Martín. Estudio descriptivo y propositivo, población de 311 docentes y 33 directivos y la muestra 75 docentes y el 100\% de los directivos. Aplicó la encuesta y el cuestionario. Concluye la necesidad de aplicar de un SGC basado en ISO 9001:2015 que brinde valor agregado en las dimensiones de capacitación y desarrollo continuo.

El tema en estudio se sustenta con las teorías aportadas por los diferentes autores. Así tenemos a Davenport y Short (1990) que definen un proceso como un conjunto de actividades y procedimientos interrelacionados para transformar datos de entrada en salida para crear valor y responder a una necesidad del entorno (Simón, 2018; p.39). Asimismo, Wolf y Harmnon, afirman 
que existe evidencia en la literatura sobre la creciente importancia que tiene la implementación de la gestión por procesos en las organizaciones (2012,pag. 40).

En este orden de ideas, Wolf y Harmon destacaron la importancia de implementar proyectos de gestión de procesos, principalmente en Europa y Estados Unidos, donde procesos forma parte de las operaciones continuas de las empresas (2012; p. 40).

La gestión por procesos BPM, es una filosofía de gestión con enfoque sistémico, son las actividades que aportan valor en los resultados, su origen data de los años 80 por los autores Geary Rummler, Alan Brache y James Harrington, y las compañías que incorporaron esta metogología fueron HP, IBM, Kodax, Ford (ISPI-Rummler 2004, pag.40)

Según Nurbanum(2013), citado por Ponce, K.(2016,p.20) ”BPM es un principio que reúne las mejores prácticas para apoyar a las empresas a lograr ventajas competitivas.

Los autores Michel Hammer y James Champy, en 1994 , tuvieron gran acogida por su libro "Reingeníera de la empresa", lo que produjo la presentación de nuevas metodologías interrelacionadas a la gestión por procesos, la reingeniería, el Total Quality Management (TQM), generando impacto en las empresas (Harmon 2004, pag 60).

Actualmente, la experiencia a lo largo de la trayectoria de logros e inconvenientes, ayudó a ordenar y mostrar mejor las buenas prácticas denominado "Bussiness Process Management" o Gestión por procesos, incorporando la mejora continua, reingeniería de procesos, consolidando la base de los modelos EFQM, ISO 9000:2000 y Six Sigma (Harmon 2004, pag 62)

El BPM, incrementa valor a la gestión pública, promueve la comunicación interna y externa, aporta en la resultados esperados, permite identificar las actividades que no aportan valor permitiendo el rediseño, así mismo controla y elimina tiempos informales y promueve la digitalización de los documentos .

En este sentido se comprende a la gestión por procesos como un conjunto de actividades destinadas a generar valor sobre las entradas para conseguir un resultado que satisfaga los requerimientos del cliente.

De la revisión bibliográfica se determinó las dimensiones de la metodología BPM basado en las siguientes fases:

Para la fase 1, caracterización y diagnóstico, tiene como finalidad identificar las necesidades de las áreas de resultados, representados por un responsable de cada área para determinar hacia donde debe orientar la gestión por procesos. 
Para la fase 2, análisis de los procesos, tiene como finalidad clasificar los procesos en operativos, estratégicos y de apoyo, con la finalidad de evaluar y alinear a las estrategias de la institución, tal como proponen Claveranne y Pascal.

Para la fase 3, mejora de los procesos, tiene por finalidad presentar los procesos en diagramas, mapas de procesos para realizar la gestión y el control respectivo, evalúa los tiempos de respuesta de los proceso .

Para la fase 4, seguimiento y control, tiene como finalidad evaluar el desempeño de los procesos, teniendo como indicadores que miden la eficiencia, eficacia y calidad de los procesos.

La gestión por proceso determina tres dimensiones en los servicios de salud, según lo planteado por Donabedian (1966), quien establece a las dimensiones de estructura, proceso y resultado y sus indicadores para evaluarla(p.665).

El Ministerio de Salud (2014, p.2) clasifica los procesos de la siguiente manera: Procesos estratégicos definida como objetivos estratégicos del centro de salud que responden a la visión de la organización. Procesos misionales que, constituyen los procesos transaccionales de las instituciones que impactan sobre la satisfacción del usuario, enlazando a los procesos a nivel estratégico y de soporte. Y Procesos de soporte, que abarcan las actividades necesarias para el correcto funcionamiento de los procesos operativos y estratégicos.

Nuestra variable gestión de los resultados, es definida por Álvarez (2012) como una herramienta de gestión dinámica y transparente centrada en el cumplimiento de objetivos estratégicos definidos en los planes de gobierno (p. 41). Asimismo, dimensiona la gestión por resultados considerando los componentes de la gestión por resultados i) Planificación estratégica, ii) Responsabilidad; iii) Monitoreo y evaluación y el Desarrollo de personal. La primera dimensión, referida a la panificación estratégica se define como “...un proceso de reflexión sistemática a nivel estratégico para establecer oportunidades que identifica la alta dirección, alcanzando los resultados buscados" (p. 51).

Asimismo, Kotler (1991; citado por Manes, 2014) indicó que la planificación estratégica define los objetivos estratégicos para el logro de la visión y oportunidades que presenta el entorno (p. 37). En la planificación estratégica se consideró dos indicadores: Planeamiento estratégico y Planeamiento operativo.

Respecto a la segunda dimensión "Responsabilidad", Álvarez lo define como "los metas que deben lograr las organizaciones en relación a su productividad (2012; p. 50). Asimismo, la tercera dimensión referida al monitoreo y evaluación, Álvarez (2012), como el "proceso de verificación de la ejecución de las actividades propuestas en el plan operativo del proyecto" (p. 65). Y a la 
evaluación como el "proceso que busca determinar los efectos del proyecto, en relación a las metas definida a nivel propósito y resultados" (p. 65). Y finalmente la cuarta dimensión detalla el desarrollo personal, tal es así que Álvarez (2012) refiere que consiste en "orientar desarrollo de las competencias y habilidades" (p. 49).

Dado que el enfoque por procesos es un modelo relacionado a la administración, su evolución histórica se origina en organizaciones pequeñas, con actividades de agricultura y comercio, el referente organizacional fueron los modelos jerárquicos de los militares tipo piramidal. Fue hasta 1776 cuando Adam Smith afirmó que "El origen de la riqueza proviene del trabajo de la nación que será productivo a mayor división del trabajo exista, debido al entorno y precios de mercado". (Adam Smith,p.66)

La administración científica de Frederick Taylor orienta a organizaciones modernas, hacia 1909 Max Weber presentó la Teoría Burocrática dando como aporte la eficiencia a partir del diseño organizacional. En 1916 Henry Fayol, introdujo la teoría clásica de la administración que enfatizaba el logro de objetivos(p.60)

En 1990 aparece formalmente el modelo de gestión por procesos, como estrategia para mejorar el resultado de la gestión organizacional. ¿Por qué la gestión por proceso?, según el autor (Harrington1993,p.12) porque la gestión de procesos presenta un enfoque sistémico de interrelación para lograr objetivos. Por ello es importante en la gestión considerar las actividades de planeación, organización, dirección y control para gestionar un proceso, otro punto de vista que permite lograr el objetivo es haciendo uso del ciclo de Deming con actividades como planear, hacer, verificar y actuar.

El modelo de procesos ISO 9000:2000, es un modelo que establece las relaciones entre marcos de procesos de gestión y los requisitos de gestión de calidad, la Cadena de Valor es un enfoque que permite establecer la estructura de una organización considerando valor en las actividades implementadas para brindar calidad en el cliente. El Modelo de Mejoramiento de procesos de Harrigton propone el rediseño de procesos como herramienta de gestión para generar valor implementando las estrategias definidas para el logro de la visión, traduce la estrategia en una seria de mediciones e indicadores. Uno de los modelos más conocido y utilizado es la propuesta del ISO TC 176, presentada en la serie de normas ISO 9000:2000, permite relacionar los procesos de gestión y requisitos de gestión de calidad, así mismo el modelo existe para establecer la estructura de los procesos de una organización y su secuencia considerando la generación de valor para el cliente y los accionistas denominado cadena de valor propuesta por Michael Porter.. 
¿Cuáles son las tendencias en la gestión de procesos?

Actualmente la gestión de procesos de negocios (BP;) mejora las operaciones a través de diferentes herramientas basadas en tecnologías de información como son: inteligencia artificial y asistentes virtuales para procesar grandes cantidades de información y reconocer patrones en muy poco tiempo. Internet de las cosas permiten que dispositivos se conecten a internet para intercambiar datos sin necesidad de una persona. Análisis de datos y Big Data permite a las organizaciones implementar sistemas predictivos basados en redes neuronales para comunicar hallazgos y recomendar planes de acción. Debido a estas tendencias, las organizaciones tendrán que revisar sus procesos actuales y abordar la digitalización.

Las entidades trabajan de manera funcional, es decir de forma vertical, con el enfoque por procesos orientamos a las organizaciones a gestionar por resultados, que nos muestra una visión horizontal de la entidad entendiendo el real funcionamiento de las entidades. En el Gráfico $\mathrm{N}^{\mathrm{o}} 01$ muestra el cambio de enfoque funcional al enfoque por procesos orientada a resultados.

\section{Gráfico №01 \\ De enfoque funcional a enfoque de procesos orientada a resultados}

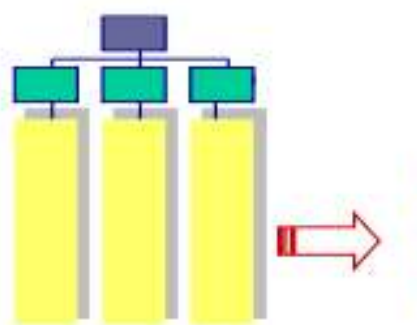

ENFOQUE FUNCIONAL

Las funciones / Áreas Gestionan la entidad

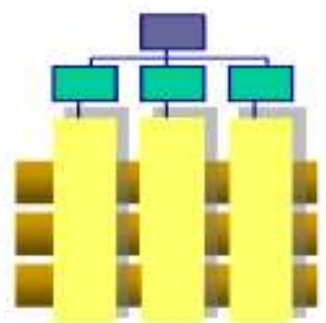

ÁREAS / PROCESOS

Se reconocen los procesos pero predominan las funciones

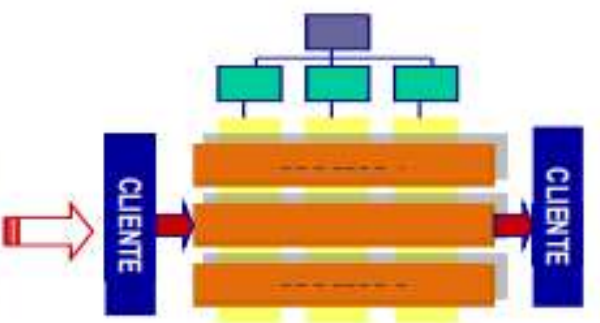

ENFOQUE DE PROCESOS

ORIENTADA A RESULTADOS

Los procesos gestionan la entidad

Fuente: Optimiso - Centro de Desarrollo Industrial (2013)

Elaboración: SGP/PCM - 2014

\section{ESTRATEGIAS METODOLÓGICAS O MATERIALES Y MÉTODOS}

La presente investigación es aplicada de enfoque cualitativo; porque permitirá proponer el modelo de gestión basado en la metodología BPM para mejorar de los resultados e indicadores. (Concytec, 2017, p.2).

La población y muestra estuvo conformada por los colaboradores del Centro de Salud de Morales, que equivale a 62 colaboradores. 
Como técnica se utilizó la encuesta y como instrumento los cuestionarios sobre aspectos de la evaluaciòn de resultados en las dimensiones de Planificaciòn estratègica, responsabilidad, monitoreo y evaluaciòn y desarrollo personal . En cuanto a la gestiòn por procesos BPM se tomó en cuenta las siguientes dimensiones: Caracterización y diagnóstico, Análisis y automatización, seguimiento y mejora de los procesos.

\section{RESULTADOS Y DISCUSIÓN}

\section{RESULTADOS}

En el presente capítulo se presentan los resultados de la investigación, los mismos que fueron procesados en gráficos.

Objetivo 1. Caracterizar la gestión actual del Centro de Salud de Morales, basado en el DS 0042013/PCM

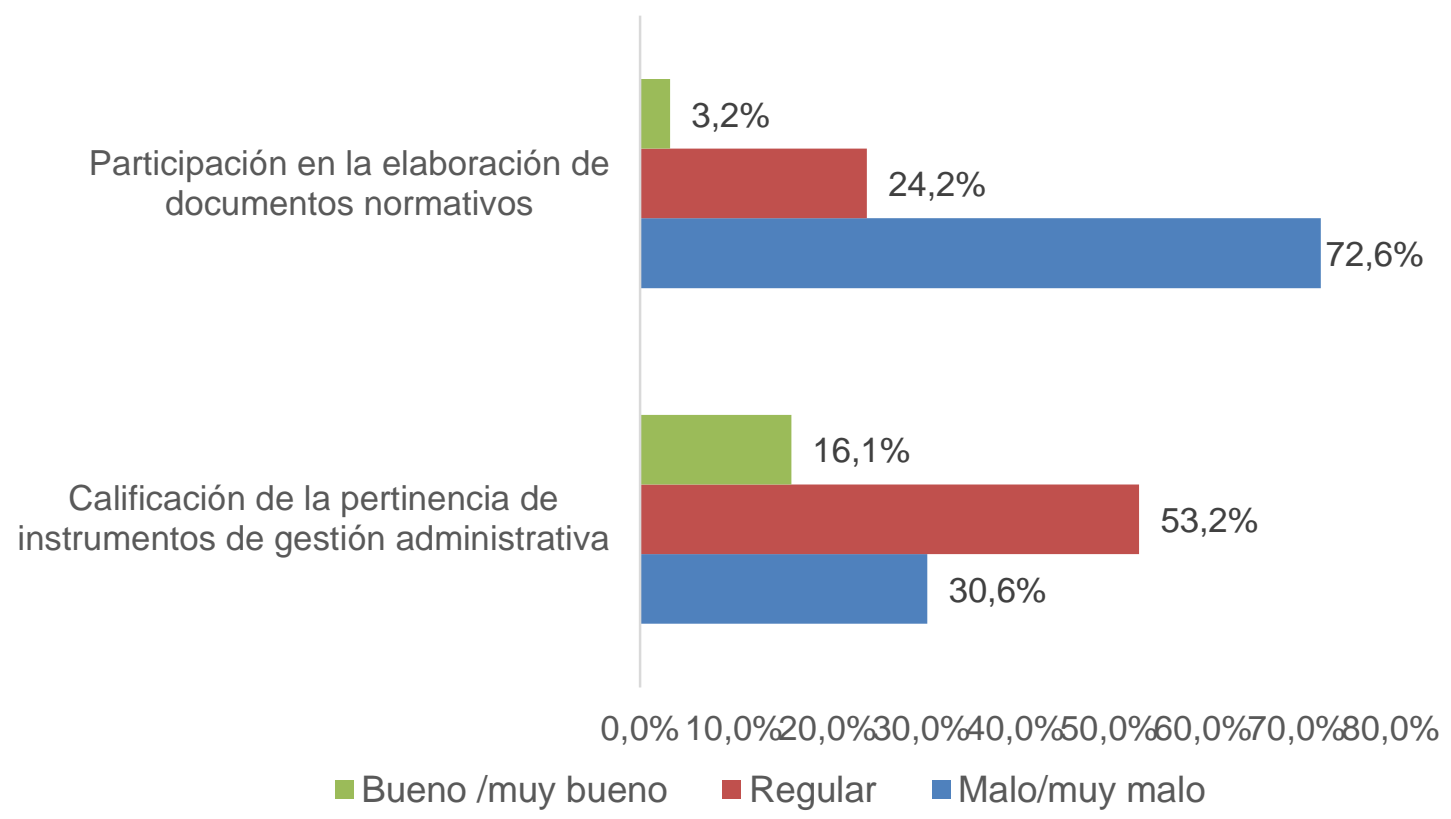

\section{Figura 1. Planificación estratégica en el Centro de Salud de Morales}

Fuente: Cuestionario aplicado a los colaboradores responsables de las áreas administrativas.

En la figura 1 observamos que el 72.6\% (45) de los colaboradores encuestados responsables de las áreas administrativas, califican como Malo y Muy malo, su participación en la elaboración de documentos normativos porque no son convocados y el 53.2\% (33) califican como regular la pertinencia de los instrumentos de gestión administrativa. 


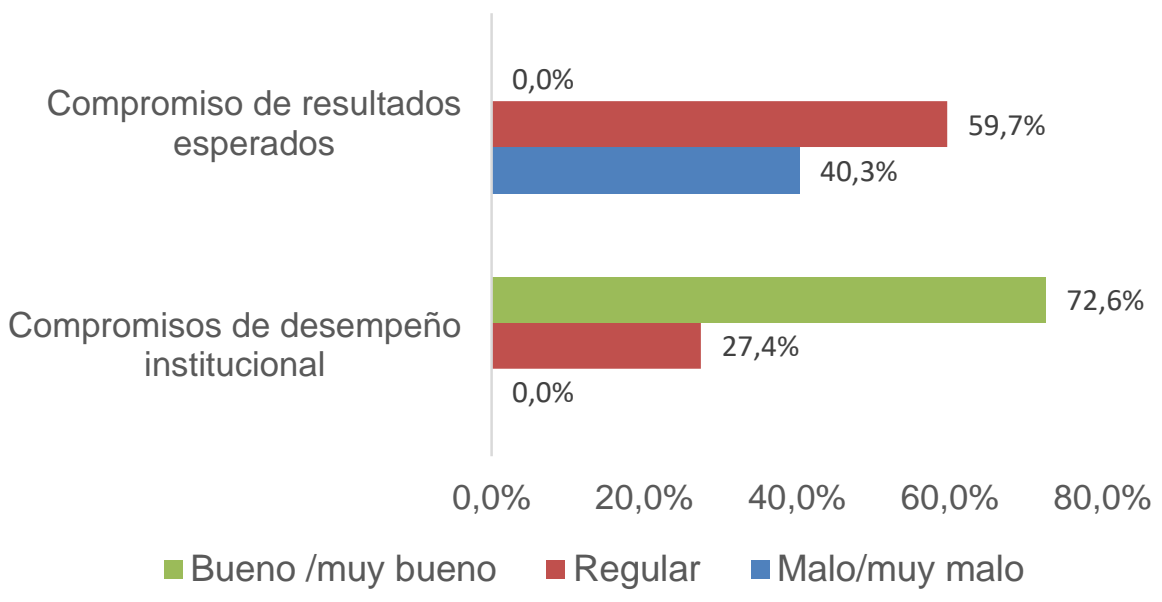

Figura 2. Responsabilidad, desempeño y compromiso institucional en el Centro de Salud de Morales

Fuente: Cuestionario aplicado a los colaboradores responsables de las áreas administrativas.

En la figura 2 observamos que el $72.6 \%$ (45) de los colaboradores encuestados responsables de las áreas administrativas, califican como Bueno/Muy Bueno, su compromiso de desempeño institucional porque se sienten identificados con la institución y el $59.7 \%$ (37) califican como Regular el compromiso de los resultados esperados por la institución porque no se socializa los instrumentos y resultados de gestión .

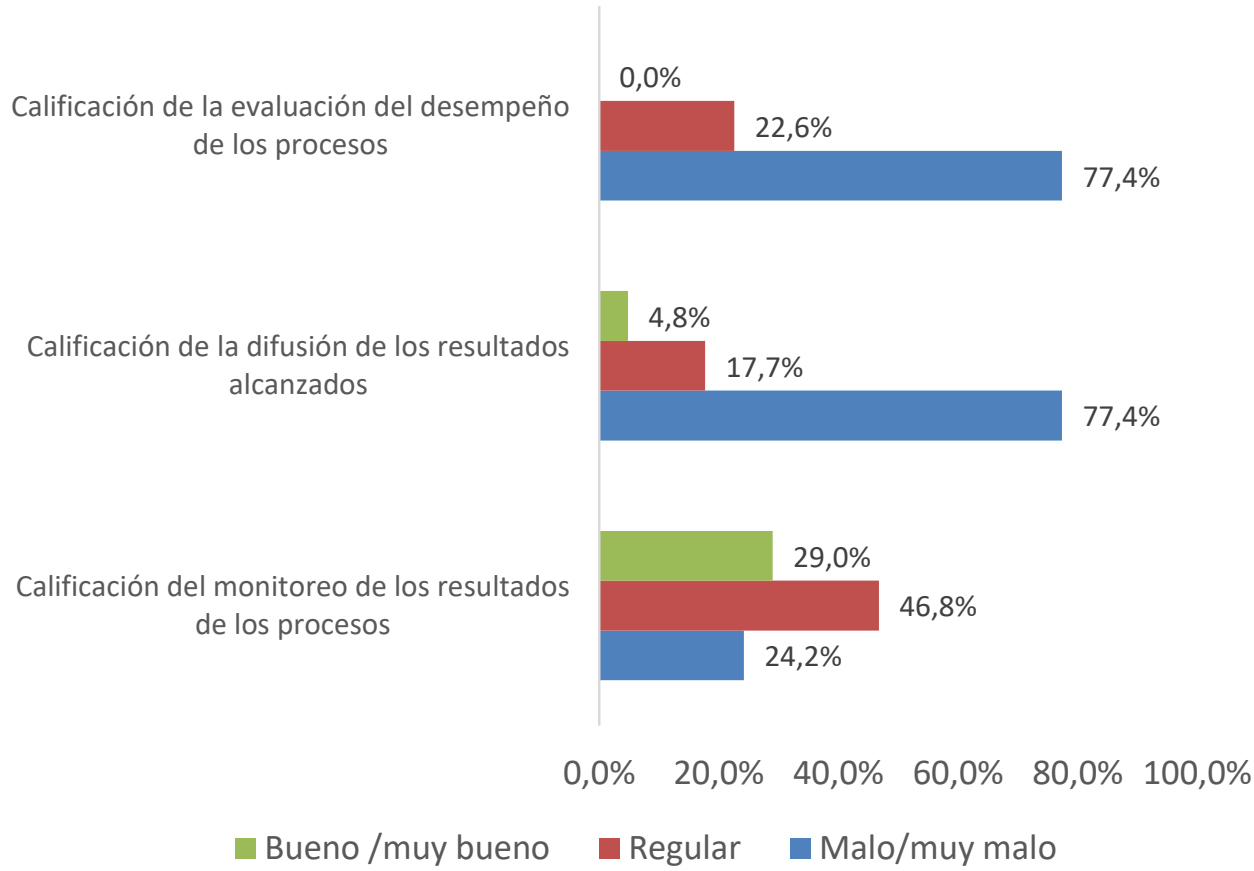


Figura 3. Monitoreo y Evaluación en el Centro de Salud de Morales

Fuente: Cuestionario aplicado a los colaboradores responsables de las áreas administrativas.

En la figura 3 observamos que el 77.4\% (48) de los colaboradores encuestados responsables de las áreas administrativas, califican como Malo/Muy Malo, la difusión de los resultados de los procesos porque no se establece un plan de gestión de calidad y el 46.8\% (29) califican como Regular el monitoreo de los resultados de los procesos y el 77.4\%(48) califica de Malo/Muy Malo el desempeño de los procesos de gestión.

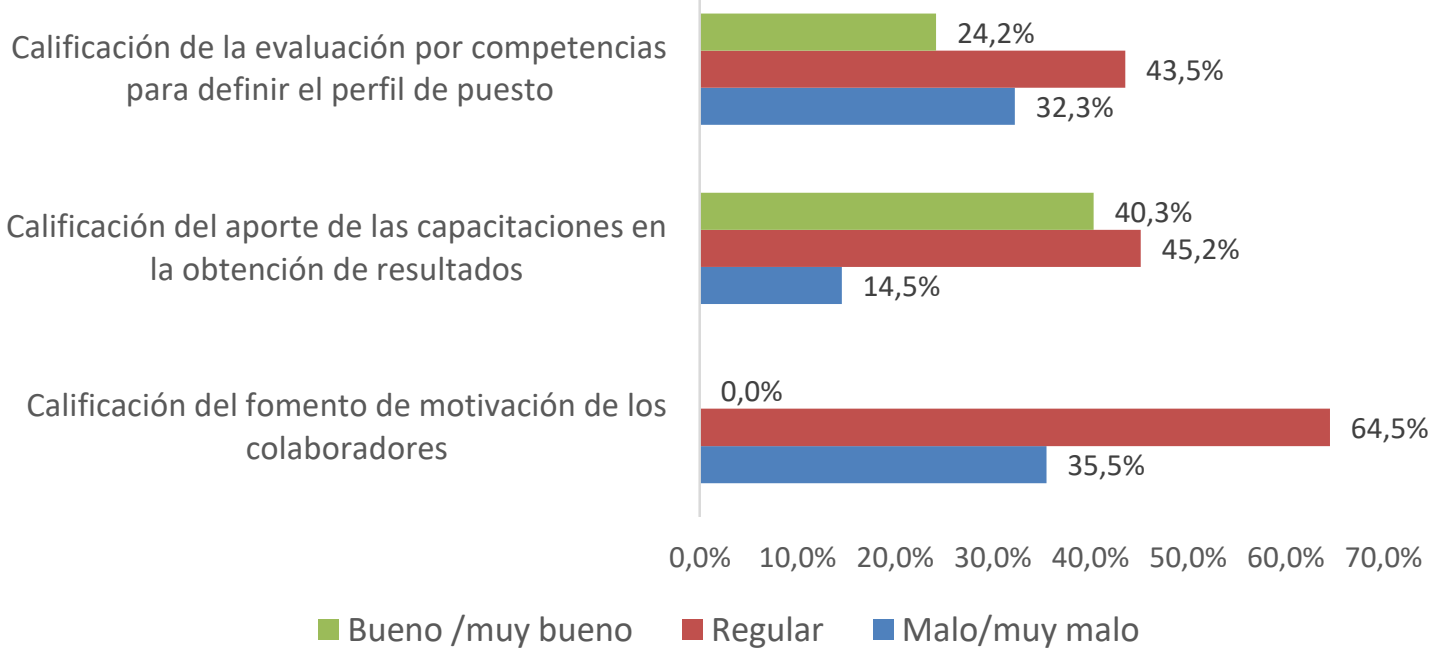

Figura 4. Desarrollo de personal en el Centro de Salud de Morales

Fuente: Cuestionario aplicado a los colaboradores responsables de las áreas administrativas.

En la figura 4 observamos que el $64.5 \%$ (40) de los colaboradores encuestados responsables de las áreas administrativas, califican como Regular, el fomento de motivación de los colaboradores esto debido a que el 43.5\% (15) califica de Regular la evaluación de competencias para definir el perfil de puestos. El 45.2\% (28) califican como Regular la calificación del aporte de las capacitaciones en la obtención de resultados porque no existe retroalimentación y seguimiento de las capacitaciones. 
Objetivo 2. Evaluar los procesos de gestión del centro de salud de Morales

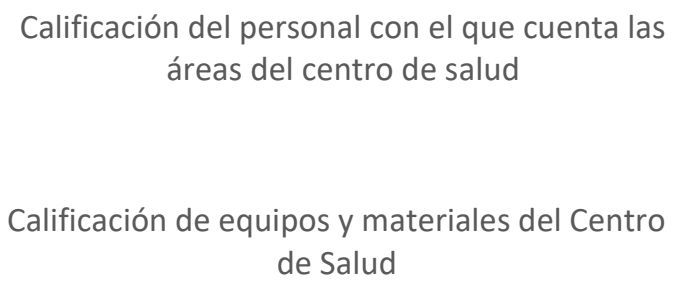

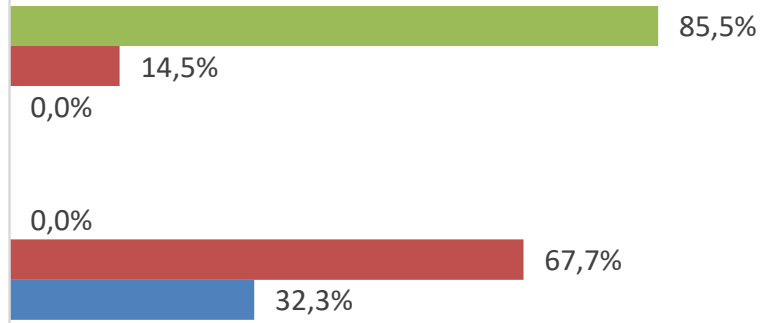

$3,2 \%$

$43,5 \%$

$53,2 \%$

0,0\% 10,0\%20,0\%30,0\%40,0\%50,0\%60,0\%70,0\%80,0\%90,0\%

Bueno / Muy bueno Regular Malo/ Muy malo

\section{Figura 5. Caracterización y diagnóstico}

Fuente: Cuestionario aplicado a los colaboradores responsables de las áreas administrativas.

En la figura 5 observamos que el $85.5 \%$ (53) de los colaboradores encuestados responsables de las áreas administrativas, califican como Bueno/Excelente, el personal con el que cuenta las áreas del centro de salud. El 67.7\% (42) califican como Regular los equipos y materiales y el 53.2\%(33) califican como Pésimo/Malo la infraestructura del centro de Salud de Morales.

\section{Calificación del análisis y modelización de los procesos de apoyo}

Calificación del análisis y modelización de los procesos estratégicos

Calificación del análisis y modelización de los procesos operativos de la institución

\section{Bueno / Muy bueno}

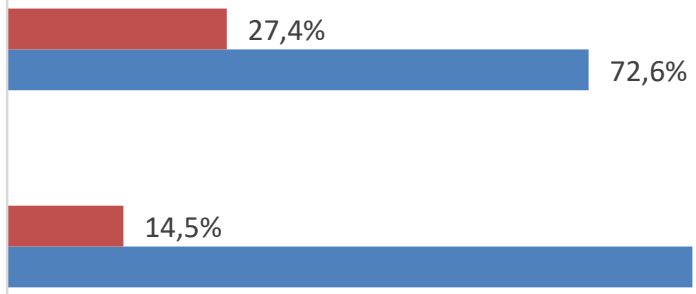

$85,5 \%$

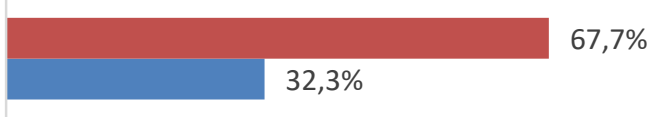

$0,0 \% 10,0 \% 20,0 \% 30,0 \% 40,0 \% 50,0 \% 60,0 \% 70,0 \% 80,0 \% 90,0 \%$

- Regular Malo/ Muy malo

Figura 6. Análisis de procesos

Fuente: Cuestionario aplicado a los colaboradores responsables de las áreas administrativas. 
En la figura 6 observamos que el $67.7 \%$ (42) de los colaboradores encuestados responsables de las áreas administrativas, califican como Regular, la calificación del análisis y modelización de los procesos operativos de la institución, el 72.6\% (45) califican como Pésimo / Malo Calificación del análisis y modelización de los procesos de apoyo y el 85.5\%(53) califica de Pésimo / Malo la calificación del análisis y modelización de los procesos estratégicos.

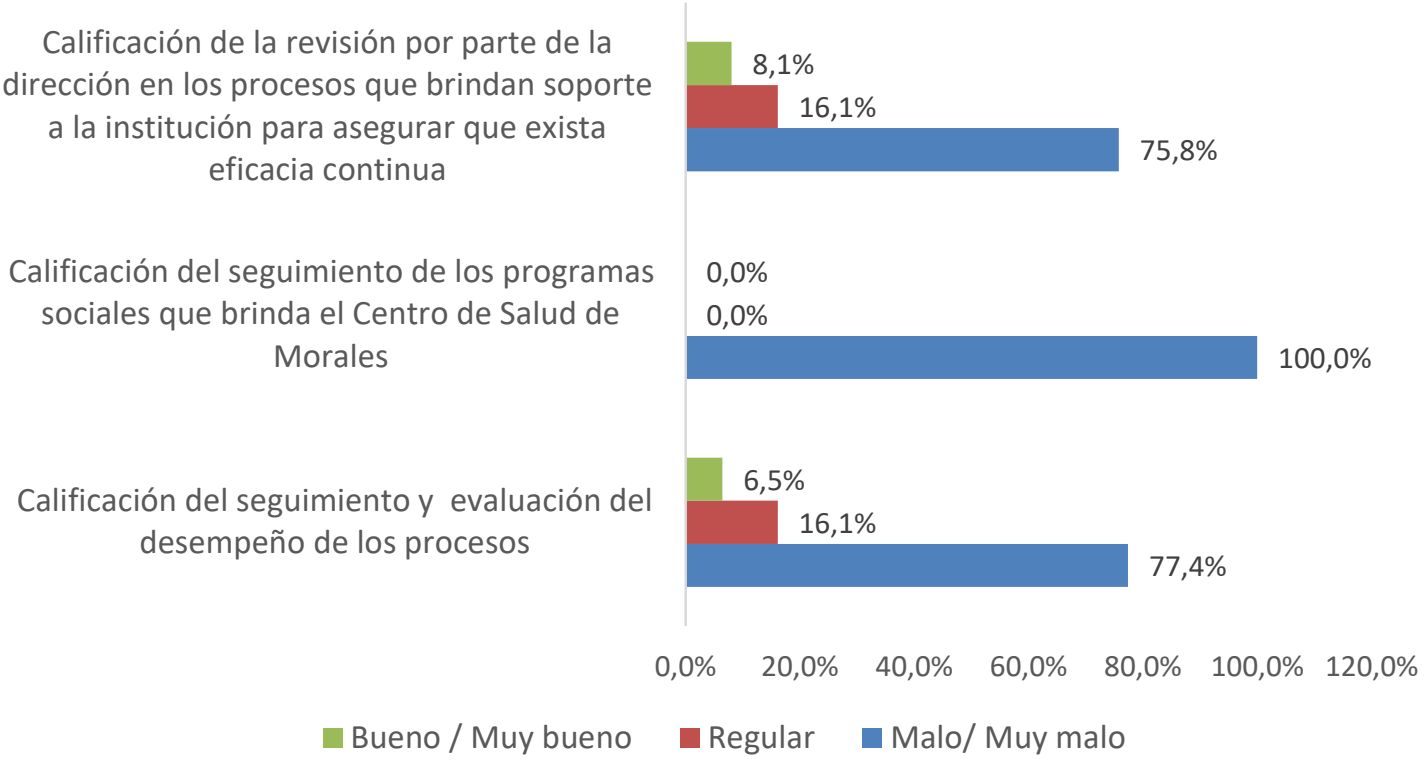

\section{Figura 7. Seguimiento}

Fuente: Cuestionario aplicado a los colaboradores responsables de las áreas administrativas.

En la figura 7 observamos que el 100\% (62) de los colaboradores encuestados responsables de las áreas administrativas, califican como Pésimo / Malo, la calificación del seguimiento de los programas sociales que brinda el Centro de Salud de Morales, el $77.4 \%$ (48) califican como Pésimo / Malo la calificación del seguimiento y evaluación del desempeño de los procesos y el 75.8\%(47) califica de Pésimo / Malo la calificación de la revisión por parte de la dirección en los procesos que brindan soporte a la institución para asegurar que exista eficacia continua. 


\section{Calificación de la selección de oportunidades de mejora con el fin de incrementar la satisfacción de los usuarios y pacientes del Centro de Salud de Morales \\ Calificación de la evaluación del nivel alcanzado}

Calificación sobre proceso de mejora contínua

Calificación sobre el manejo de guías de práctica clínica y planes estandarizados por los trabajadores

Calificación del proceso de simplificación

administrativa realizada por la institución

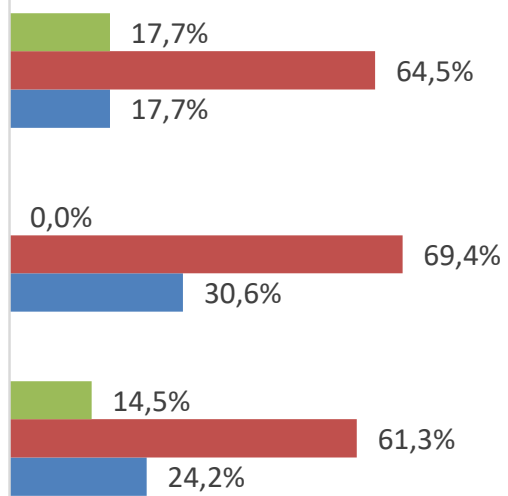

$3,2 \%$

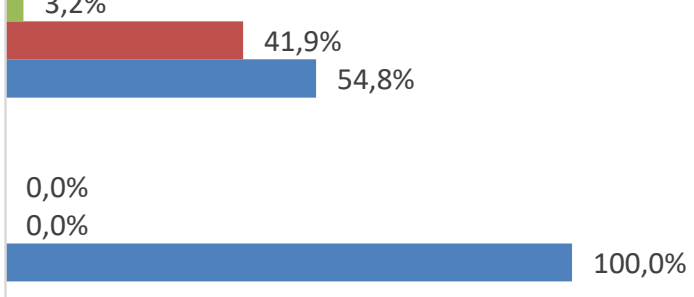

\section{$0,0 \% \quad 20,0 \% \quad 40,0 \% \quad 60,0 \% \quad 80,0 \% \quad 100,0 \% \quad 120,0 \%$}

- Regular Malo/ Muy malo

Figura 8. Mejora de los procesos

Fuente: Cuestionario aplicado a los colaboradores responsables de las áreas administrativas.

En la figura 8 observamos que el 100\% (62) de los colaboradores encuestados responsables de las áreas administrativas, califican como Pésimo / Malo, la Calificación del proceso de simplificación administrativa realizada por la institución, el 54.8\% (34) califican como Pésimo / Malo la calificación sobre el manejo de guías de práctica clínica y planes estandarizados por los trabajadores, el 61.3\%(38) califica de Regular el proceso de mejora continua, el 69.4\%(43) califica de Regular la evaluación del nivel alcanzado y el 64.5\%(40) califica de Regular la calificación de la selección de oportunidades de mejora con el fin de incrementar la satisfacción de los usuarios y pacientes del Centro de Salud de Morales.

\section{DISCUSIÓN}

Para el análisis del objetivo específico 1, se realizó la caracterización de la gestión actual del Centro de Salud de Morales a 62 colaboradores, donde se observa: 
En la dimensión Planificación estratégica, el 72.6\% de los colaboradores encuestados responsables de las áreas administrativas, califican como "malo y muy malo", su participación en la elaboración de documentos normativos, debido a que los colaboradores no son convocados en un $60 \%$ y califican como "regular" la pertinencia de los instrumentos de gestión administrativa en un 53.2\%. Datos similares fueron encontrados por Ramos, J., García, J., Cid, V. (2018), quienes indican que los gestores deben mantener actualizado los planes existentes, y elaborar los planes faltantes, aplicando estrategias para preservar el compromiso y participación de los colaboradores y agentes del entorno. Así mismo lo muestra Ríos(2018) donde, el 47.2\% indicaron que existe un nivel deficiente respecto a la gestión en la dimensión planificación estratégica. Al respecto en la investigación realizada por Fonseca, Hernández, Medina y Nogueira (2014) en la tesis Relevancia de la planificación estratégica en la gestión pública; concluye que gracias a una adecuada planificación estratégica se permitió identificar que los modelos de gestión consultados y que para su desarrollo se pueden inferir pasos comunes e igualmente útiles en el entorno público, en la cual los resultados descriptivos son semejantes a la presente investigación.

En la dimensión Responsabilidad, desempeño y compromiso institucional el $72.6 \%$ de los colaboradores encuestados responsables de las áreas administrativas, califican como Bueno/Muy Bueno, su compromiso de desempeño institucional porque se sienten identificados con la institución, existe una actitud emprendedora, se adecua fácilmente a los cambios que ocurren dentro de la institución, son personas innovadoras y asumen riesgos con el fin de lograr resultados positivos de gestión, respectivamente. Datos similares fueron encontrados por Ríos(2018), quién afirma que el $48 \%$ de los encuestados indica que existe un nivel moderado respecto a la responsabilidad.

En la dimensión Monitoreo y Evaluación el 77.4\% (48) de los colaboradores encuestados responsables de las áreas administrativas, califican como Malo/Muy Malo, la difusión de los resultados de los procesos porque no se establece un plan de gestión de calidad y el $46.8 \%$ (29) califican como Regular el monitoreo de los resultados de los procesos y el 77.4\%(48) califica de Malo/Muy Malo el desempeño de los procesos de gestión. . Datos similares fueron encontrados por Ríos(2018), quién afirma que el $31.1 \%$ de los encuestados indica que existe un nivel deficiente respecto al monitoreo y evaluación. 
En la dimensión desarrollo de personal, el 64.5\% de los colaboradores encuestados de las áreas administrativas, califican como regular, el fomento de motivación de los colaboradores, debido a que las estrategias de evaluación de competencias para definir el perfil de puestos es regular en un $43 \%$. . Datos similares fueron encontrados por Ríos(2018), quién afirma que el 34\% de los encuestados indica que existe un nivel deficiente respecto al desarrollo de personal. Asimismo en la investigación realizada por Sánchez (2016) también concluye que es importante que los directivos realicen la motivación a su personal, como en toda investigación la motivación es el factor clave en un mejor desempeño de la organización, asimismo los resultados son semejantes respecto a la administración pública.

En la dimensión caracterización y diagnóstico el $67.7 \%$ de los colaboradores califican como Regular los equipos y materiales, la calificación del análisis y modelización de los procesos operativos de la institución, el 72.6\% (45) califican como Pésimo / Malo Calificación del análisis y modelización de los procesos de apoyo y el 85.5\%(53) califica de Pésimo / Malo la calificación del análisis y modelización de los procesos estratégicos. En la dimensión seguimiento el 100\% de los colaboradores encuestados responsables de las áreas administrativas, califican como Pésimo / Malo, la calificación del seguimiento de los programas sociales que brinda el Centro de Salud de Morales, el 77.4\% (48) califican como Pésimo / Malo la calificación del seguimiento y evaluación del desempeño de los procesos y el 75.8\%(47) califica de Pésimo / Malo la calificación de la revisión por parte de la dirección en los procesos que brindan soporte a la institución para asegurar que exista eficacia continua.

En la dimensión mejora de los procesos, el 100\% de los colaboradores encuestados responsables de las áreas administrativas, califican como Pésimo / Malo, la Calificación del proceso de simplificación administrativa realizada por la institución, el $54.8 \%$ califican como Pésimo / Malo la calificación sobre el manejo de guías de práctica clínica y planes estandarizados por los trabajadores, el 61.3\%(38) califica de Regular el proceso de mejora continua, el 69.4\% califica de Regular la evaluación del nivel alcanzado y el 64.5\% califica de Regular la calificación de la selección de oportunidades de mejora con el fin de incrementar la satisfacción de los usuarios y pacientes del Centro de Salud de Morales. De igual manera, los autores Hammer y Champy (año), quienes establecen que las nuevas metodologías interrelacionadas a la gestión por procesos, generan impacto 
positivo en las empresas. Actualmente, la experiencia, ayuda a ordenar y mostrar mejor las buenas prácticas denominado "Bussiness Process Management" o Gestión por procesos, incorporando la mejora continua, reingeniería de procesos, consolidando la base de los modelos EFQM, ISO 9000:2000 y Six Sigma (Harmon 2004, pag 62)

\section{CONCLUSIONES O CONSIDERACIONES FINALES}

Existe una calificación de 53\% parcialmente regular de los resultados de la gestión actual en las dimensiones de planificación estratégica, responsabilidad, desempeño y compromiso institucional, monitoreo y evaluación y desarrollo de personal, frente al $85 \%$ califica de pésimo los procesos de las áreas administrativas.

Se evaluó los procesos de la gestión del Centro de Salud de Morales, de acuerdo a las dimensiones de caracterización y diagnóstico, análisis de procesos, seguimiento, mejora de procesos. Donde los gestores deben hacer esfuerzos para organizarse con normas, políticas y estrategias de comunicación y estimulación interpersonal. La caracterización constituye la vía para familiarizarse con la actividad esencial de la organización y tener una idea inicial de hacia dónde debe apuntar la gestión de sus procesos.

El modelo de gestión por procesos basado en la metodología BPM se fundamenta en los resultados obtenidos del diagnóstico situacional de la gestión de la gestión del Centro de Salud de Morales y de la teoría de la Gestión por procesos BPM y la tecnología de la información y comunicaciones como herramienta de soporte de BPM. La aplicación de un grupo de estas herramientas en instituciones hospitalarias matanceras, asociadas a un procedimiento para la inserción de la gestión por procesos refleja, en los resultados obtenidos, su utilidad y pertinencia para la mejora de los procesos hospitalarios y su contribución, por ende, a la calidad del servicio que ellas brindan.

\section{LISTA DE REFERENCIAS}

Barrios, K., Contreras J.(2019) La Gestión por Procesos en las Pymes de Barranquilla: Factor Diferenciador de la Competitividad Organizacional (artículo científico). Facultad de Ingeniería, Universidad Simón Bolívar, Barranquilla-Colombia. Consultado en: https://scielo.conicyt.cl/scielo.php?script=sci_arttext\&pid=S071807642019000200103 $\& \operatorname{lng}=$ en\&nrm=iso\&tlng 
Cantón, I. (2010), "Introducción a los Procesos de Calidad”. Revista Iberoamericana sobre Calidad, Eficacia y Cambio en Educación (REICE). Vol. 8 No 5, pp. 45-53. 2016. ISSN: 1696-4713., recuperado en : https://revistas.uam.es/index.php/reice/article/view/4723

Carrasco, H., Farroñay D.(2017) Diseño de procesos aplicando business process management para la empresa DHL @utos S.A.C.(Trabajo de investigación) Universidad Nacional Pedro Ruiz Gallo. Perú Disponible en http://repositorio.unprg.edu.pe/bitstream/handle/UNPRG/1017/BC-TES5782.pdf?sequence $=1 \&$ isAllowed $=\mathrm{y}$

Chàvez, R.(2017) Modelo de Gestión por Procesos Apoyado Con Las Tecnologías de Información y Comunicaciones para el Help Desk en una Institución Pública, Universidad Nacional Federico Villarreal, recuperado de : http://repositorio.ucsp.edu.pe/bitstream/UCSP/15240/1

Cuba, A. (2018), Aplicación del Modelo de Proceso de Negocios (BPM) y su efecto en la gestión de calidad del laboratorio clínico del hospital Cayetano Heredia (Trabajo de investigación grado de maestría) Universidad César Vallejo. Consultado en: http://repositorio.ucv.edu.pe/bitstream/handle/UCV/21325/Cuba_DAE.pdf?sequence=1 \&isAllowed=y

Delgado, A.,Hernàndez, A.(2016) Cuba. Generalización de la gestión por procesos como plataforma de trabajo de apoyo a la mejora de organizaciones de salud (artículo científico), Universidad de Matanzas Camilo Cienfuegos de Cuba, Rev. Gerenc. Polít. Salud. 2016; 15(31): 66. Consultado https://revistas.javeriana.edu.co/index.php/gerepolsal/article/view/18220

Donabedian (2011). "Donabedian Y La Calidad de La Atencion." Universidad del Zulia Maracaibo, Venezuela www.uca.edu.ar/.../anexogdrfioravanti-avedis-donabedian-ylaca.

Estela, R. (2016). Gestión por procesos, disciplina para diseñar la estructura organizacional del Ministerio de Salud del Perú - 2014 (Tesis de pregrado en Administración de Empresas). Universidad de Piura. Perú. Recuperado de https://pirhua.udep.edu.pe/bitstream/handle/11042/2630/AEL 007.pdf?sequence=1

Estrella, A. (2012). Gestión por Procesos: Alternativa para mejorar la prestación de Servicios de Salud. Artículo Científico Biblioteca digital ecuatoriana. Recuperado de http://www.bibliotecasdelecuador.com/Record/ir-:21000-6635 
Fernández M. (1999), Gestión de la función calidad en los servicios médicos asistenciales de segundo nivel (Tesis Doctoral en Ciencias Técnicas). Las Villas, Cuba, Universidad Central "Marta Abreu" de las Villas, Santa Clara, Cuba

Fernández, R. (2017). Incidencia de la reforma del Estado en la Modernización de la Gestión Pública en las Universidades Nacionales, caso UNSA-2015 Arequipa. (Tesis para optar Grado de Doctor en Ciencias Empresariales). Universidad Nacional de San Agustín. Perú. Recuperado http://repositorio.unsa.edu.pe/bitstream/handle/UNSA/2460/COfeferc.pdf?seque nce=1

Gonzalez, E. (2014), Despliegue de la calidad en la gestión de los procesos sustantivos de instituciones de educación superior cubanas (Tesis Doctoral). Universidad Central Marta Abreu de las Villas, Santa Clara, Cubas. Consultado en:https://dspace.uclv.edu.cu/bitstream/handle/123456789/12298/Ebir\%20qfd.pdf?sequ ence $=1 \&$ isAllowed $=\mathrm{y}$

Guzman, P., Montalvo F. Carvallo, E. \& Raymundo, C., Implementation of a process management model and inventory control to increase the level of service in the after-sales area of industrial equipment. Universidad Peruana de Ciencias Aplicadas. Perú , recuperado en : http://dx.doi.org/10.18687/LACCEI2019.1.1.147

Hak, M., Andrlic, B.,Sostar, M. (2018) Using information technology in business process reengineering: Case study of tax administration Croatia (artículo científico) Croatia. Recuperado de: https://www.scopus.com/record/display.uri?eid=2-s2.0$\underline{85059853074 \& \text { origin }=\text { resultslist } \& \text { sort }=\text { plf- }}$

$\mathrm{f} \& \mathrm{src}=\mathrm{s} \& \mathrm{st} 1=\mathrm{Bussine}+$ Process + Management $\& \mathrm{st} 2=\& \mathrm{sid}=\mathrm{f} 6 \mathrm{~b} 15 \mathrm{a} 9 \mathrm{a} 6 \mathrm{~d} 637 \mathrm{f} 3 \mathrm{f} 26 \mathrm{c} 559 \mathrm{e} 0$ 846d111f\&sot=b\&sdt=b\&sl=42\&s=TITLE-ABS-

$\underline{\text { KEY\%28Bussines+Process+Management\%29\&relpos }=1 \& \text { citeCnt }=0 \& \text { searchTerm }}$

Hernández, A., Delgado, A. (2016) Generalización de la gestión por procesos como plataforma de trabajo de apoyo a la mejora de organizaciones de salud (artículo científico). Universidad de Matanzas Camilo Cienfuegos, Cuba. Recuperado de: https://www.redalyc.org/pdf/545/54549363016.pdf

http://dx.doi. org/10.11144/Javeriana.rgyps15-31.ggpp

Huaman,J. , Villanueva, G.(2016), Propuesta Business Process Management Para La Facultad De Ingeniería De La Upagu, 2016, (Trabajo de investigación), recuperado en: http://repositorio.upagu.edu.pe/handle/UPAGU/100 
Medina, A. (2018). Procedimiento para la gestión por procesos: métodos y herramientas de apoyo (artículo científico). Universidad de Matanzas Cuba. Consultado en https://scielo.conicyt.cl/pdf/ingeniare/v27n2/0718-3305-ingeniare-27-02-00328.pdf

http://dx.doi.org/10.4067/S0718-33052019000200328

Ministerio de Salud. (2017). Decreto Supremo Nº 008-2017-SA. Reglamento de Organización y Funciones del Ministerio de Salud. Recuperado de ftp://ftp2.minsa.gob.pe/normaslegales/2017/rof_2017.pdf

Navarro, N.( 2017) Gestión por procesos y su relación con la calidad de Servicio en el Área de Obstetricia del Centro e Salud Del Distrito De Andahuaylas, 2016(Trabajo de investigación) Universidad Nacional José María Arguedas, Consultado en http://repositorio.unajma.edu.pe/bitstream/handle/123456789/306/Nancy_Tesis Bachiller_2017.pdf?sequence $=1 \&$ isAllowed $=\mathrm{y}$

Navarro, F. (2016). Introducción a la Gestión por Procesos. Revista digital INESEM. Recuperado de https://revistadigital.inesem.es/gestion-integrada/gestion-porprocesos/.

Norma Internacional ISO 9001. (2015). Sistema de gestión de la calidad-Requisitos (5ed.).Suiza.Recuperadodehttp://www.imre.uh.cu/wordpress/wpcontent/uploads/ 2015/06/ISO_9001_2015.pdf

Organización Mundial de la Salud [OMS] (2009). Estadísticas Sanitarias Mundiales - 2009. Recuperado de http://www. who.int/entity/gho/publications/world_health_statistics/ ES_WHS09_Full.pdf

Paredes, M., Pailiacho, V., Robayo, D., (2017). Optimización de los Procesos de Mesa de Ayuda: Un Enfoque desde ITIL. (Artículo científico). Universidad Católica del Ecuador. Consultado en: $\quad$ https://www.scopus.com/record/display.uri?eid=2-s2.0$\underline{85058816363 \& \text { origin }=\text { resultslist } \& \text { sort }=\text { plf- }}$

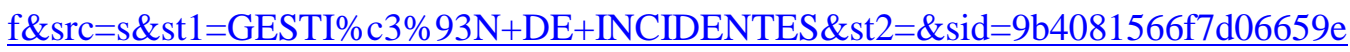
64e514dcf206b8\&sot=b\&sdt=b\&sl=36\&s=TITLE-ABSKEY\%28GESTI\%c3\%93N+DE+INCIDENTES\%29\&relpos=6\&citeCnt=0\&searchTer $\underline{m}=$ 
Palomino, G. (2018). Modelo de gestión por procesos para mejorar la evaluación tributaria en las municipalidades provinciales. (Artículo científico). Universidad Nacional de San Martín, Tarapoto, Perú. Recuperada de http://www.tesisenred.net/bitstream/handle/10803/31762/ TMAFV.pdf

Pascual, C. (2015) Definición y estabilización de los procesos I. procesos de gestión y soporte en un servicio de Urología (artículo científico) Facultativo Especialista de Area. Unidad de Urología. Hospital Universitario Infanta Cristina Parla. Madrid (España). Consultado en: https://www.scopus.com/record/display.uri?eid=2-s2.0$\underline{84945243710 \& \text { origin }=\text { resultslist } \& \text { sort }=\text { plf- }}$ $\mathrm{f} \& \mathrm{src}=\mathrm{s} \& \mathrm{st} 1=\mathrm{gesti} \% \mathrm{c} 3 \% \mathrm{~b} 3 \mathrm{n}+$ procesos $\& n l \mathrm{o}=\& \mathrm{n} l \mathrm{r}=\& \mathrm{nls}=\& \mathrm{sid}=0 \mathrm{~cd} 4 \mathrm{~d} 1 \mathrm{fd} 11 \mathrm{ed} 4 \mathrm{a} 8 \mathrm{e} 2 \mathrm{de}$ bcb3f94efe997\&sot=b\&sdt=sisr\&sl=31\&s=TITLE-ABS-

KEY\%28gesti\%c3\%b3n+procesos\%29\&ref=\%28\%28gesti\%c3\%b3n+por+proceso\%29 \%29+AND+\%28gesti\%c3\%b3n+por+procesos\%29\&relpos=29\&citeCnt=2\&searchTer $\underline{\mathrm{m}}=$

Prado, W.(2018), Nivel de desarrollo de la gestión por procesos en los hospitales de Nivel II de Lima Metropolitana - Ministerio de Salud ( trabajo de investigación), Universidad Nacional Mayor De San Marcos, recuperado en: https://www.researchgate.net/publication/315815023_La_gestion_por_procesos _en_hospitales

Ponce, K. (2016) Propuesta de implementación de gestión por procesos para incrementar los niveles de productividad en una empresa textil. UPC: Lima, Perú. (Trabajo de investigación) recuperado

en https://repositorioacademico.upc.edu.pe/bitstream/handle/10757/620981/Tesis+ Textil+S.A.C.++ Katherine + Ponce + Herrera.pdf;jsessionid=914FB6201FA3158B54CA7BA18C $\underline{6 \mathrm{E} 3 \mathrm{AD} 9}$ ? sequence $=1$

Quillama, L. (2008). Proceso de Modernización del Estado. Presidencia de Consejo de Ministros - Secretaría de Gestión Pública. Programa de capacitaciones. Lima Perú.Recuperado de Www.gobiernodigital.gob.pe/capacitaciones/Programas_docu/24/Programa_124. pdf 
Ramos, J. \& García J. \& Cid V.(2018) Diseño de un marco de trabajo dirigido por modelos y soportado por herramientas para mejorar la gestión de guías clínicas y procesos asistenciales (artículo científico) Universidad de Sevilla, España. Consultado en

https://www.scopus.com/record/display.uri?eid=2-s2.0-

85071753345\&origin=resultslist\&sort=plf-

$\mathrm{f} \& \mathrm{src}=\mathrm{s} \& \mathrm{st} 1=\mathrm{gesti} \% \mathrm{c} 3 \% \mathrm{~b} 3 \mathrm{n}+$ procesos $\&$ nlo $=\& \mathrm{nlr}=\& \mathrm{nl}=\& \mathrm{sid}=0 \mathrm{~cd} 4 \mathrm{~d} 1 \mathrm{fd} 11 \mathrm{ed} 4$ a8e2debcb3f94efe997\&sot=b\&sdt=sisr\&sl=31\&s=TITLE-ABS-

KEY\%28gesti\%c3\%b3n+procesos\%29\&ref=\%28\%28gesti\%c3\%b3n+por+proc eso $\% 29 \% 29+\mathrm{AND}+\% 28 \mathrm{gesti} \% \mathrm{c} 3 \% \mathrm{~b} 3 \mathrm{n}+$ por + procesos $\% 29 \&$ relpos=9\&citeCnt= 0\&searchTerm

Schmiedel, T. , Recker, J., Brocke, J. (2019) The relation between BPM culture, BPM methods, and process performance: Evidence from quantitative field studies, (artículo científico), University of Applied Sciences and Arts Northwestern Switzerland, recuperado de: https://www.sciencedirect.com/science/article/abs/pii/S0378720618302805

Santa María T.(2018), Gestión por procesos y su relación con la asistencia técnica contable para los usuarios del Centro de Servicios de Atención al Usuario CONECTAMEF (Tesis de maestría) Universidad César Vallejo Departamento de San Martín. Consultado en: http://repositorio.ucv.edu.pe/bitstream/handle/UCV/31403/Santa\%20Mar\%C3\% ADa_FT.pdf? sequence $=1 \&$ isAllowed $=y$

Rahman, W. (2015). Proposed it financial management process using ITIL (TI infrastructure library) for Port Company in Indonesia. Journal Scense Indonesia. Obtenido en : http://ppm.telkomuniversity.ac.id/home/proposed-it-financialmanagement-process-using-itil-it-infrastructure-library-for-port-company-inindonesia/

Robles, L. \& Díaz, P.(2017) Aplicando La Gestión Por Procesos En El Sector Salud Del Perú, Rev Acad Peru Salud 24(1). Artículo de Revisión. Recuperado de: http://bvs.minsa.gob.pe/local/MINSA/4156.pdf 
Rodriguez, M. (2013) en su investigación Manejo de herramientas de gestión y percepción del cuidado de Enfermería en hospitales de Chachapoyas, Perú,(artículo científico) Universidad Nacional Toribio Rodríguez de Mendoza de Amazonas, Chachapoyas. Consultado en: http://www.scielo.org.mx/pdf/eu/v11n1/v11n1a2.pdf

The Association of Business Process Management Professionals International . (2013). Guía de referencia de la Asociación de Profesionales de BPM. ABPMP

Samaniego D.(2018) Gestión administrativa y calidad de servicio en el área de neonatología del Hospital II - 2 Tarapoto, 2018(Tesis de maestría) Universidad César Vallejo. Consultado en http://repositorio.ucv.edu.pe/bitstream/handle/UCV/31862/Samaniego_HDM.pd $\mathrm{f}$ ? sequence $=1 \&$ isAllowed $=\mathrm{y}$

Sepúlveda, H. (s.f.). BPM se está posicionando en el mundo como el modelo de gestión organizacional por excelencia. Colombia. Obtenido de http://www.clubbpm.com/Noticias/art00112.htm

Silva, V. (2013) Aplicación de gestión por procesos, como herramienta de apoyo al mejoramiento del Hospital dr. Eduardo Pereira (Trabajo de investigación grado de maestría) Universidad de Chile. Consultado en:http://bibliodigital.saludpublica.uchile.cl:8080/dspace/bitstream/handle/12345 6789/375/Tesis_Viviana+Silva.pdf;jsessionid=7B7F3D53880139DF0D59DB43 BDED5287? sequence $=1$

Vallejos, V. (2018) Sistema de Gestión de Calidad según ISO 9001:2015 para mejorar procesos de capacitación, Universidad Nacional de San Martín - Tarapoto, 2018(tesis doctoral) Escuela De Posgrado Programa Académico De Doctorado En Gestión Pública Y Gobernabilidad Universidad César Vallejo. Consultado en : http://repositorio.ucv.edu.pe/handle/UCV/37461?show=full

Zapa, E. (2014). Impacto de la Gestión por Procesos en la Innovación de las Organizaciones. Instituto Tecnológico Metropolitano. Cintex, 19: 23-37. 
http://www.pascualbravo.edu.co/cintex/index.php/cintex/article/view/145/145

\section{Libros}

Aranaz, J., Mira J., Lorenzo S, Barbeito E.(2003) La valoración de los resultados generales de la actividad asistencial en los servicios de cirugía. Cirugía Española, recuperado en: https://www.researchgate.net/publication/242604287_La_gestion_por_procesos _asistenciales_integrados

Banco Mundial. 2008. Performance-Informed Budgeting in Latin America. Experiences and Opportunities. Washington, D.C.: Banco Mundial., recuperado de : https://publications.iadb.org/publications/spanish/document/La-gesti\%C3\%B3npara-resultados-en-el-desarrollo-Avances-y-desaf\%C3\%ADos-en-

Am\%C3\%A9rica-Latina-y-el-Caribe.pdf

Beltrán, J. (2013) Guía para una Gestión basada en Procesos. Instituto Andaluz de Tecnología. España (2013), recuperado en : https://www.euskadi.eus/web01s2ing/es/contenidos/informacion/bibl_digital/es_documen/adjuntos/Guia\%20par a\%20una\%20gestion-basada-procesos.pdf

BonitaSoft.com. (2013). La Guía Definitiva de BPMN 2, recuperado en : https://es.bonitasoft.com/biblioteca/la-guia-definitiva-de-bpmn2

Brocke, J. (2013), Manual de bpm : gestão de processos de negócio, Portuguese. Recuperado de: http://eds.a.ebscohost.com/eds/detail/detail?vid=0\&sid=2db3dbba6222-47f6-8dae-f608a8cb3187\%40sdc-vsessmgr02\&bdata=Jmxhbmc9ZXMmc210ZT11ZHMtbG12ZQ\%3d\%3d\#AN=edsmib.00 $\underline{0004378 \& \mathrm{db}=\mathrm{edsmib}}$

Hitpass, B. (2017). BPM: Business Process Management: Fundamentos y Conceptos de Implementación 4a Edición actualizada y ampliada. Dr. Bernhard Hitpass. Recuperado en $\quad: \quad$ https://www.amazon.com/BPM-Management-Fundamentos-ConceptosImplementaci\%C3\%B3n/dp/1546667970 
García, R., García M.(2010) La Gestión para Resultados en el Desarrollo, Avances y desafíos en América Latina y el Caribe, Banco Interamericano de desarrollo, recuperado de: https://publications.iadb.org/publications/spanish/document/La-gesti\%C3\%B3n-pararesultados-en-el-desarrollo-Avances-y-desaf\%C3\%ADos-en-Am\%C3\%A9rica-Latinay-el-Caribe.pdf

Goetsch, D.L. (2014) Quality management for organizational excellence, 40, Pearson, Upper Saddle River, New Jersey, USA, Recuperado en : https://www.pearsonhighered.com/assets/preface/0/1/3/5/0135577322.pdf

Hernández, A. (2003) Enfoque por procesos, la cadena vital de la organización. En: Industrial. Vol. XXIV, $\mathrm{N}^{\circ}$ 1.recuperado en : https://goo.gl/wZcayo

Ministerio de Salud (2013) Indicadores de Gestión y Evaluación Hospitalaria, Para Hospitales, Institutos Y Diresa, recuperado de: http://bvs.minsa.gob.pe/local/MINSA/2739.pdf

J. Bravo Carrasco. "Gestión avanzada de Procesos". Editorial Evolución S. A. Santiago de Chile, Chile. , recuperado en : https://pdfslide.net/download/link/gestion-avanzada-deprocesos-de-j-bravo

Morris, E., Serida, J., Yamakawa, P.(2011), Estudio de madurez de las empresas peruanas en la gestión de procesos: BPM 2011. Esan ediciones. Recuperado de: https://www.esan.edu.pe/publicaciones/libros/2011/estudio-de-madurez-de-lasempresas-peruanas-en-la-gestion-de-procesos-bpm-2011/

Concytec, (2017), REGLAMENTO DE CALIFICACIÓN, CLASIFICACIÓN Y REGISTRO DE LOS INVESTIGADORES DEL SISTEMA NACIONAL DE CIENCIA, TECNOLOGÍA E INNOVACIÓN TECNOLÓGICA - REGLAMENTO RENACYT, recuperado en : https://portal.concytec.gob.pe/images/noticias/Manual_del_Reglamento_RENACYT_1. pdf

Hitpass B. Business Process Management. Fundamentos y Conceptos de Implementación. $3^{\text {a }}$. Edición. Universidad Técnica Federico Santa María. Santiago de Chile (2014)

LINEAMIENTOS PARA LA IMPLEMENTACIÓN DE LA GESTIÓN POR PROCESOS EN LAS ENTIDADES DE LA ADMINISTRACIÓN PÚBLICA EN EL MARCO DEL D.S. $\mathrm{N}^{\circ}$ 004-2013-PCM - POLÍTICA NACIONAL DE MODERNIZACIÓN DE LA GESTIÓN PÚBLICA AL 2021 
Ministerio de Salud (2014) Mapa de Procesos del Ministerio de Salud, Oficina General de Planeamiento y Presupuesto - OGPP

Ministerio de Salud(2016), Arquitectura Empresarial del Ministerio de Salud. Arquitectura de Negocios - Modelo de Información. Versión 1.2. Chile, recuperado en : https://www.gob.cl/ministerios/ministerio-de-salud/

Alvarez, C. , Lara, A. Torres, C. , Covarrubias, L. (2014) Modelo de Evaluación De Programas De Salud, Secretaría de Salud y Bienestar Social del Estado de Colima México. Recuperado http://www.saludcolima.gob.mx/images/documentos/ModeloDeEvaluacionDePrograma sDeSalud.pdf

ONGEI.(2017). Decreto Supremo de Política Nacional de Gobierno Electrónico 2013-2017. Obtenido en: http://www.pcm.gob.pe/normaslegales/2013/DS-081-2013-PCM.pdf

ISACA. (2017). Instituto de Gobernanza de TI (ITGI).Recuperado de http://www.isaca.org/About-ISACA/IT-GovernanceInstitute/Pages/default.aspx

Presidencia de Consejo de Ministros, (2017), Política Nacional de Modernización del Estado al 2021. Aprobado por D.S. Nº04- 2013-PCM. Lima, Perú

Sampieri, H., Fernandez, C., \& Baptista, P. (2014). Metodología de la Investigación. (H. Mc Graw, Ed.) (Sexta). Ciudad de México: Mc Graw, Hill.

http://www.minsa.gob.pe/transparencia/index.asp?op=601\# 